\title{
Obesidade e sua associação com agravamento da COVID-19: uma revisão sistemática
}

\section{com meta-análise}

Obesity and its association with aggravation of COVID-19: a systematic review with meta-analysis

Obesidad y su asociación con agravación de COVID-19: una revisión sistemática con metaanálisis

\section{Resumo}

Objetivo: Analisar e compilar estudos que relacionam a obesidade com complicações da COVID-19. Métodos: Realizou-se uma revisão sistemática com meta-análise, utilizando a estratégia PECOS: Paciente - obesos; Exposiçãopessoas com COVID-19; Comparação - não obesos; "Outcome" - agravamento do quadro clínico; e "Studies"estudos observacionais. As buscas foram realizadas em 16/06/2020 no PubMed, Lilacs e SciELO. A qualidade metodológica foi avaliada pela escala STROBE e os dados foram sintetizados de maneira qualitativa. Modelos de meta-análise foram realizados para necessidade de Unidade de Terapia Intensiva (UTI), Ventilação Mecânica Invasiva (VMI) e mortalidade. Resultados: Foram avaliados 24 estudos, retrospectivos, publicados em 2020, de diferentes países. O tamanho das amostras foi de 53 a 3615 pacientes, de faixa etária variável, de etnias diversas, sem restrição de sexo e internados em centros de saúde. Na avaliação de qualidade 8/11 atingiram pontuação de $77 \%$ em A ou B. Em relação aos fatores metabólicos, os estudos mostraram que alterações propiciadas pelo tecido adiposo em excesso favorecem a desregulação dos níveis de interleucinas, desencadeando um quadro infeccioso crônico. A metaanálise da frequência de UTI foi de $27 \%$ entre obesos, necessidade de VMI foi de $34 \%$ e mortalidade foi de $14 \%$. Conclusão: Constatou-se que a obesidade é um preditor para o agravamento da COVID-19. Portanto, indivíduos obesos necessitam de uma abordagem precoce bem como um atendimento especial em caso de contaminação.

Palavras-chave: Coronavírus; Sobrepeso; Mortalidade; Obesidade; SARS-CoV-2; Unidade de terapia intensiva; Ventilação mecânica.

\begin{abstract}
Objective: To analyze and compile studies linking obesity with complications from COVID-19. Methods: A systematic review with meta-analysis was carried out, using the PECOS strategy: Patient - obese; Exhibition - people with COVID-19; Comparison - not obese; "Outcome" - worsening of the clinical condition; and "Studies" observational studies. Searches were performed on 06/16/2020 in PubMed, Lilacs and SciELO. Methodological quality was assessed using the STROBE scale and data were qualitatively synthesized. Meta-analysis models were performed for Intensive Care Unit (ICU) need, Invasive Mechanical Ventilation (IMV) and mortality. Results: 24 retrospective studies published in 2020 from different countries were evaluated. The sample size ranged from 53 to 3615 patients, of varying age, of different ethnicities, without gender restrictions and admitted to health centers. In the $8 / 11$ quality assessment, they reached $77 \%$ in A or B. Regarding metabolic factors, studies showed that alterations caused by excess adipose tissue favor the deregulation of interleukin levels, triggering a chronic infectious condition. The meta-analysis of the frequency of ICU was $27 \%$ among obese, need for IMV was $34 \%$ and mortality was $14 \%$. Conclusion: It was found that obesity is a predictor for the worsening of COVID-19. Therefore, obese individuals need an early approach as well as special care in case of contamination.
\end{abstract}


Keywords: Coronavirus; Overweight; Mortality; Obesity; SARS-CoV-2; Intensive care unit; Mechanical ventilation.

\begin{abstract}
Resumen
Objetivo: Analizar y recopilar estudios que relacionen la obesidad con las complicaciones del COVID-19. Métodos: Se realizó una revisión sistemática con metaanálisis, utilizando la estrategia PECOS: Paciente - obeso; Exposición personas con COVID-19; Comparación - no obeso; "Resultado": empeoramiento de la condición clínica; y "Estudios" - estudios observacionales. Las búsquedas se realizaron el 16/06/2020 en PubMed, Lilacs y SciELO. La calidad metodológica se evaluó mediante la escala STROBE y los datos se sintetizaron cualitativamente. Se realizaron modelos de metanálisis por necesidad de Unidad de Cuidados Intensivos (UCI), Ventilación Mecánica Invasiva (VMI) y mortalidad. Resultados: Se evaluaron 24 estudios retrospectivos publicados en 2020 de diferentes países. El tamaño de la muestra varió de 53 a 3615 pacientes, de diversa edad, de diferentes etnias, sin restricciones de género, e ingresados en centros de salud. En la evaluación de calidad del 8/11, alcanzaron el 77\% en A o B. En cuanto a los factores metabólicos, los estudios mostraron que las alteraciones provocadas por el exceso de tejido adiposo favorecen la desregulación de los niveles de interleucina, desencadenando una enfermedad infecciosa crónica. El metaanálisis de la frecuencia de UCI fue del $27 \%$ entre los obesos, la necesidad de VMI fue del $34 \%$ y la mortalidad fue del 14\%. Conclusión: Se encontró que la obesidad es un predictor del empeoramiento de COVID-19. Por lo tanto, las personas obesas necesitan un abordaje temprano, así como un cuidado especial en caso de contaminación.
\end{abstract}

Palabras clave: Coronavirus; Exceso de peso; Mortalidad; Obesidad; SARS-CoV-2; Unidad de terapia intensiva; Ventilacion mecanica.

\title{
1. Introdução
}

Os coronavírus são uma grande família de vírus Coronaviridae. Recebem esse nome devido aos espinhos em forma de coroa que podem ser vistos em sua superfície com microscópio eletrônico (Netto et al., 2020). Esses vírus foram identificados pela primeira vez em meados da década de 1960, como patógenos que causam infecções respiratórias e intestinais em seres humanos e em animais. A organização Mundial da Saúde (OMS), em dezembro de 2019, confirmou o surgimento de uma nova cepa do coronavírus (nome temporário 2019-nCoV), que teve seus primeiros casos diagnosticados com Síndromes Respiratórias leves e graves, na cidade de Wuhan, província de Hubei, na República Popular da China. Aproximadamente um mês depois (11 de fevereiro de 2020), a OMS, renomeou o vírus para SARS-CoV-2, responsável por causar a doença infecciosa COVID-19(OPAS/OMS Brasil, 2020).

O vírus SARS-Cov-2 tem como estrutura um nucleocapsídeo, uma fita simples de RNA como material genético e algumas proteínas, como as proteínas spikes, as quais dão formato de coroa para o vírus. Essas proteínas precisam encontrar um receptor específico nas células humanas para infecta-las. Esse receptor é chamado de ECA-2 (enzima conversora de angiotensina 2) e ele costuma estar presente nas células epiteliais, incluindo o epitélio pulmonar, coração e rins (Netto et al., 2020).

Existem indícios de que há uma associação entre o agravamento da COVID-19 e doenças crônicas como hipertensão, diabetes e a obesidade (de Oliveira, Lucas \& Iquiapaza, 2020). Esta quase triplicou em todo o mundo desde 1975, pelo que foi caracterizada como uma pandemia. Em 2016, 39\% da população adulta mundial (mais de 1,9 bilhões de pessoas) estavam acima do peso e $13 \%$ eram obesos (mais de 650 milhões de pessoas), enquanto que em 2018, 40 milhões de crianças com menos de 5 anos estavam acima do peso ou obesas (World Health Organization, 2020). Esses percentuais são ainda maiores na America Latina, haja vista que quase $60 \%$ da população tem sobrepeso e $25 \%$ são consideradas obesas. Esses valores triplicaram nas ultimas quatro décadas, colocando a America Latina em segundo lugar nas taxas de obesidade do mundo, perdendo apenas para a América do Norte segundo as Nações Unidas e a Organização para a Cooperação e Desenvolvimento Econômico (OCDE) (Nações Unidas Brasil, 2019).

O excesso de peso é um grande problema para a saúde pública pois gera elevados gastos que poderiam ser evitados. Isso se deve ao fato do mesmo desencadear diversas comorbidades como: diabetes, dislipidemia, doença hepática não gordurosa, hipertensão arterial sistêmica, doenças ortopédicas, fibrose cística, além de dificultar os movimentos respiratórios. Além disso, 
a obesidade é uma doença complexa de etiologia multifacetada, com sua própria fisiopatologia, comorbidades e de capacidades desabilitantes (Luiz Do Prado, Lofrano, Oyama \& Damaso, 2009). Representa um estado de inflamação de baixo grau, com vários produtos inflamatórios secretados diretamente pelo tecido adiposo (Michalakis \& Ilias, 2020). Nesse cenário destacamse a IL-6,IL-1, IL-2, TNF $\alpha$ (tumor necrosis factor $\alpha$ ), leptina, adiponectina e adipsina, que formam a "tempestade de citocinas" responsável pela desregulação imunológica e por consequência o agravamento do quadro clínico dos pacientes infectados por SARS-Cov-2.

De acordo com a Federação Mundial da Obesidade, "as condições relacionadas à obesidade parecem piorar o efeito do COVID-19 (SARS-CoV-2)", uma vez que há um ambiente hormonal desfavorável. Devido a isso, é possível perceber que embora os níveis de leptina estejam aumentados, a ação da leptina por si só é reduzida, de maneira análoga à ação da insulina em pacientes com DM2, sugerindo que a resistência à leptina poderia agravar o resultado dos pacientes durante a COVID-19, (Inês Grenha, Alves, Ribeiro \& Cavaco, 2013). Dessa forma, a leptina exerce efeitos negativos na maturação, desenvolvimento e função das células B, além de alterações dos linfócitos, inibição do CD8 + e a resposta das células T. Desse modo, o ambiente imunológico observado na obesidade, que em circunstâncias normais atuaria contra o vírus, acaba favorecendo sua replicação e proliferação.

Há diversos estudos sendo produzidos que descrevem a ação do coronavírus no organismo de indivíduos obesos. No entanto, há necessidade de síntese das evidências produzidas no sentido de contribuir com governos e entidades na tomada de decisão para melhor controle da doença também neste grupo. Sendo assim, a questão norteadora da pesquisa foi: pacientes obesos, com COVID-19 tem maior propensão de desenvolver a forma mais grave da doença?

Diante da questão o objetivo da presente revisão foi analisar e compilar estudos que relacionam a obesidade e as complicações da COVID-19, bem como entender como sua fisiopatologia corrobora para a necessidade de UTI, VMI e mortalidade.

\section{Metodologia}

Trata-se de uma revisão sistemática com meta-análise, registrada no PROSPERO sob numero CRD42020196311, baseada nas diretrizes metodológicas de elaboração de revisão sistemáticas e meta-análise de estudos observacionais, comparativos sobre fatores de risco e prognósticos (Brasil. Ministério da Saúde. Secretaria de Ciência, 2014). Guiada pela seguinte pergunta de pesquisa "Pacientes obesos, com COVID-19, tem maior propensão de desenvolver a forma mais grave da doença? Sustentada na estratégia PECOS: Paciente - obesos, Exposição- pessoas com COVID-19, Comparação - pacientes não obesas, o "Outcome" - agravamento do quadro clínico e "Studies"- foram incluídos os estudos observacionais comparativos.

\section{Critérios de inclusão}

Foram incluídos estudos observacionais ou de coorte, bem como, estudos que abordaram características epidemiológicas, clinicas, metabólicas e físicas da COVID-19 e sua associação com a obesidade, com ou sem resultados quantitativos.

Foram incluídos pacientes obesos e não obesos (o IMC foi usado como uma forma de medir o grau de obesidade) que apresentaram resultado positivo para COVID-19 ao serem submetidos a um teste sorológico, teste rápido ou swab. Não levouse em consideração a faixa etária dos pacientes, sexo e etnia. 


\section{Critérios de exclusão}

Foram excluídos relatos de casos, ensaios clínicos e outras revisões sistemáticas. Além de estudos que não apresentaram uma relação clara e objetiva entre COVID-19 e obesidade, tais como: artigos referentes a comorbidades, regimes nutricionais, aspectos não COVID-19 de cirurgia bariátrica, coronavírus em animais, medicamentos no tratamento da obesidade, mecanismos da doença COVID-19 sem considerar a obesidade.

\section{Busca e identificação dos artigos}

As bases eletrônicas pesquisadas foram PubMed, Lilacs e Scielo. Sendo que a base de dados LILACS é compostas por referências bibliográficas de documentos científicos e técnicos da área da saúde referentes à América Latina e Caribe. A base SciELO é uma biblioteca eletrônica que abrange uma coleção selecionada de periódicos científicos brasileiros. A base PubMed foi desenvolvida e é mantida pela Biblioteca Nacional de Medicina (NLM®) dos Estados Unidos. A busca na literatura foi realizada em 16 de junho de 2020, no entanto não delimitou-se ano de publicação e idioma, conforme as diretrizes metodológicas de revisão sistemática e meta-análise. Para a busca, foram realizadas combinações com os operadores boleanos "and" e "or", utilizando os descritores controlados Overnutrition or Abdominal obesity or Obesity or Overweight or Over fat or Fat or Fatty or Obese or Body weight or Body mass index and Coronavírus or Covid-19 or SARS-Cov-2 de acordo com os Descritores em Ciências da Saúde (DeSC) e Medical Subject Headings (MeSH).

Os estudos obtidos em todas as combinações e bases foram selecionados utilizando o MENDELEY (Singh, 2010) por meio da eliminação das duplicatas, identificação, seleção e avaliação da elegibilidade.

\section{Seleção dos estudos}

A seleção dos estudos foi realizada de maneira independente por dois revisores (A.C. e J.P.) e as discordâncias foram decididas por um terceiro. Inicialmente os artigos foram selecionados após a leitura do título e os que atenderam os critérios de elegibilidade e que tiveram consenso entre os dois revisores, foram selecionados. Em seguida, foram lidos os resumos e os artigos escolhidos nesse segundo momento, foram analisados na íntegra para inclusão ou exclusão da revisão.

\section{Sumarização dos dados}

Os estudos incluídos tiveram seus dados sumarizados por dois avaliadores, utilizando para isso o formulário de extração de dados modificado do instrumento proposto pelas diretrizes metodológicas utilizadas (Brasil. Ministério da Saúde. Secretaria de Ciência, 2014). Identificando artigo, ano, autores, características e tamanho da amostra, intervenção, sinais/sintomas, resultado, (n) UTI, (n) sem UTI, (n) VMI, tempo de internação na UTI e (n) mortalidade para obesos e não obesos. Além disso, marcadores metabólicos foram extraídos selecionados de acordo com as necessidades da pesquisa, pois são fatores que influenciam na fisiopatologia das doenças, COVID-19 e obesidade.

Os resultados quantitativos de necessidade de UTI, VMI e mortalidade foram extraídos conforme a disponibilidade nos artigos. Para isso os pacientes dos estudos foram classificados em quatro categorias de avaliação de risco (resultado obeso presente, resultado obeso ausente, resultado não obeso presente e resultado não obeso ausente) de acordo com os desfechos já citados.

\section{Avaliação da qualidade metodológica}

A qualidade foi avaliada usando a escala Strengtheningn the reporting of observational studies in epidemiology (STROBE), traduzida e adaptada para o idioma português, com recomendações para melhorar a qualidade dos estudos 
observacionais (estudos de coorte, caso-controle e estudos seccionais). Trata-se de uma lista de verificação de 22 itens para check-list de informações, com recomendações sobre o que deveria ser incluído em uma descrição mais precisa e completa de estudos observacionais, possibilitando uma avalição crítica dos achados. O risco de viés de publicação não pode ser avaliado pelo teste de Egger pois a quantidade de estudos utilizados na meta-analise foi menor do que o mínimo necessário para se aplicar esse teste.

\section{Síntese dos resultados}

Os resultados foram sintetizados de maneira qualitativa através de uma avaliação crítica dos mesmos. Em cada estudo, os desfechos obtidos (necessidade de UTI, VMI e mortalidade) foram descritos em termos de método e principais resultados, a fim de verificar a consistência e a validade dos mesmos.

Com os resultados quantitativos, foram realizados modelos de meta-análise com o método de variação inversa para resultados binários (risco relativo) para necessidade de UTI, VMI e mortalidade associado à obesidade. A heterogeneidade entre os resultados foi avaliada pelo teste $\mathrm{I}^{2} \mathrm{e}$, quando significativos, foram utilizados modelos de efeitos aleatórios e, quando não significativos, modelos de efeitos fixos. Estas análises foram realizadas com o pacote "meta" no ambiente R (Balduzzi \& Rücker, 2019)

\section{Resultados}

Identificou-se nas bases eletrônicas 1942 artigos, dos quais 1.290 foram excluídos por serem referências duplicadas. Após a exclusão, 652 estudos foram lidos e avaliados pelo título. Depois de realizada a leitura do título e resumo, 603 artigos não atenderam aos critérios de elegibilidade, sendo que 49 artigos foram lidos na íntegra. Em seguida, foram excluídos 25 artigos restando 24 artigos dos quais 11 havia dados quantitativos. Os artigos não escolhidos foram excluídos pelas seguintes razões: eram relatos de casos, ensaios clínicos e outras revisões sistemáticas. Além de estudos que não apresentaram uma relação clara e objetiva entre COVID-19 e obesidade, tais como: artigos referentes a comorbidades, regimes nutricionais, aspectos não-COVID de cirurgia bariátrica, coronavírus em animais, medicamentos no tratamento da obesidade, mecanismos da doença COVID -19 sem considerar a obesidade. O total dos artigos incluídos que atenderam aos critérios de inclusão foram 24, estes permitiram a análise qualitativa. Sendo possível a análise quantitativa para 11 artigos (Figura 1). 
Figura 1 - Fluxograma da seleção de evidências, baseada nas diretrizes do PRISMA.
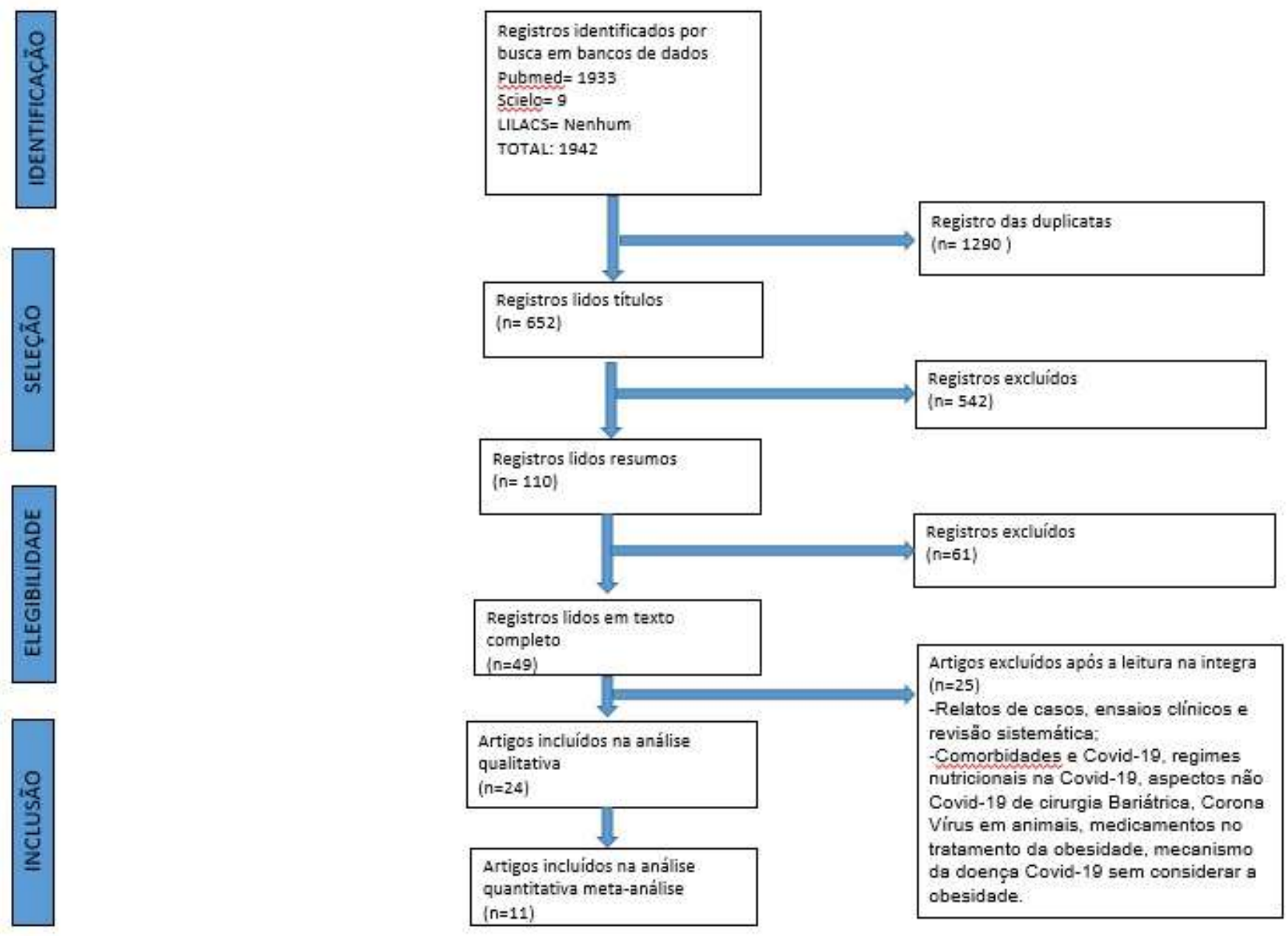

Fonte: Autores.

Na Tabela 1, estão descritos os estudos incluídos e suas características qualitativas. Os 24 estudos incluídos foram retrospectivos, todos publicados no ano de 2020 e de diferentes países, tais como Estados Unidos, China, Itália, França e Singapura. O tamanho da amostra dos estudos variou entre 53 a 3615 pacientes com teste positivo para COVID-19, obesos e não obesos, de faixa etária que variou de adolescentes a idosos com idade superior a 80 anos, de etnias diversas, sem restrição de sexo e que foram internados em centros de saúde. Dentre os estudos selecionados alguns são multicêntricos (Kalligeros et al., 2020). Ainda, na Tabela 1, está relatada a avaliação de qualidade que demonstrou que a pontuação de 8 dos 11 artigos quantitativos, na escala STROBE, atingiram pontuação de $77 \%$ em A ou B (atendeu completamente e parcialmente os quesitos). Já na Figura 2, foram descritos os desfechos dos artigos incluídos que, em sua grande maioria, apresentaram o agravamento da doença, necessidade de UTI, VMI e mortalidade (Hajifathalian et al., 2020). Para isso, fatores metabólicos e físicos foram abordados para sustentar suas teorias. 
Tabela 1 - Estudos incluídos e suas características qualitativas

\begin{tabular}{|c|c|c|c|}
\hline Autor, ano & $\begin{array}{l}\text { (1) Design do Estudo } \\
\text { (2) Acompanhamento }\end{array}$ & $\begin{array}{l}\text { (1) Tamanho da amostra } \\
\text { (2) Características da amostra } \\
\text { (3) Intervenção e comparação }\end{array}$ & $\begin{array}{c}\text { Avaliação de } \\
\text { qualidade } \\
\text { (STROBE) }\end{array}$ \\
\hline Zhang et al,. 2020 & $\begin{array}{l}\text { (1) Quantitativo, retrospectivo; (2) } \\
49 \text { dias }\end{array}$ & $\begin{array}{l}\text { (1) 53; (2) Jovens identificados com Covid-19; (3) Sobreviventes e falecidos após internação } \\
\text { hospitalar }\end{array}$ & $\begin{array}{l}\mathrm{A}-73 \% \\
\mathrm{~B}-15 \%\end{array}$ \\
\hline Kass et al., 2020 & $\begin{array}{l}\text { (1) Quantitativo, retrospectivo; (2) } \\
1 \text { dia }\end{array}$ & (1) 265 ; (2) Indivíduos identificados com Covid -19 & $\begin{array}{l}\mathrm{A}-41 \% \\
\mathrm{~B}-21 \%\end{array}$ \\
\hline Lighter et al., 2020 & $\begin{array}{l}\text { (1) Quantitativo, retrospectivo; (2) } \\
30 \text { dias }\end{array}$ & $\begin{array}{l}\text { (1) } 3615 \text {; (2) Indivíduos que sinalizaram positivos para o Covid-19; (3) OB e não-OB em pronto- } \\
\text { socorro, internados em hospital e UTI }\end{array}$ & $\begin{array}{l}\mathrm{A}-59 \% \\
\mathrm{~B}-25 \%\end{array}$ \\
\hline Kalligeros et al., 2020 & $\begin{array}{l}\text { (1) Quantitativo, retrospectivo; (2) } \\
49 \text { dias }\end{array}$ & $\begin{array}{l}\text { (1) } 103 \text {; (2) Adultos consecutivos ( } \geq 18 \text { anos), positivos para a Covid-19, hospitalizados; (3) OB e } \\
\text { não OB que precisaram de internação na UTI e pacientes que não precisaram }\end{array}$ & $\begin{array}{l}\mathrm{A}-58 \% \\
\mathrm{~B}-19 \%\end{array}$ \\
\hline Moriconi et al., 2020 & $\begin{array}{l}\text { (1) Quantitativo, retrospectivo; (2) } \\
31 \text { dias }\end{array}$ & (1) 100 ; (3) OB e não OB, necessidade de UTI, aporte de $\mathrm{O} 2$ e tempo de uso e mortalidade & $\begin{array}{l}\mathrm{A}-87 \% \\
\mathrm{~B}-3 \%\end{array}$ \\
\hline Kassir et al., 2020 & $\begin{array}{l}\text { (1) Quantitativo, retrospectivo; (2) } \\
27 \text { dias }\end{array}$ & (1) 112 ; (2) Internados com infecção por COVID-19; (3) Sobreviventes e não sobreviventes & $\begin{array}{l}\mathrm{A}-34 \% \\
\mathrm{~B}-3 \%\end{array}$ \\
\hline Hajifathalian et al., 2020 & $\begin{array}{l}\text { (1) Quantitativo, retrospectivo; (2) } \\
37 \text { dias }\end{array}$ & $\begin{array}{l}\text { (1) 770; (2) Indivíduos com Covid-19 de idade média de } 63,5 \text { anos, IMC médio de } 29 \mathrm{~kg} / \mathrm{m} 2 \text { e } 61 \% \\
\text { eram masculinos; (3) Pacientes com COVID-19 OB e não OB, desenvolvimento da forma grave da } \\
\text { doença, UTI, VMI e óbito entre os grupos }\end{array}$ & $\begin{array}{l}\mathrm{A}-90 \% \\
\mathrm{~B}-0 \%\end{array}$ \\
\hline Ong et al., 2020 & (1) Quantitativo, retrospectivo & $\begin{array}{l}\text { (1) } 182 \text {; (2) Asiáticos com Covid-19, idade }<60 \text { anos, IMC } \geq 25 \text { e IMC }<25 \text {; (3) Relação entre os } \\
\text { pacientes de IMC }<25 \text { e } \geq 25 \text { e O2 e VMI }\end{array}$ & $\begin{array}{l}\mathrm{A}-39 \% \\
\mathrm{~B}-29 \%\end{array}$ \\
\hline
\end{tabular}


Research, Society and Development, v. 10, n. 15, e350101522965, 2021

(CC BY 4.0) | ISSN 2525-3409 | DOI: http://dx.doi.org/10.33448/rsd-v10i15.22965

Palaiodimos et al, 2020

Busetto et al, 2020

Simonnet et al., 2020
(1) Quantitativo, retrospectivo; (2)

21 dias

(1) Quantitativo, retrospectivo; (2)

20 dias;

(1) Quantitativo, retrospectivo; (2)

38 dias;
(1) 200; (2) Pacientes consecutivos admitidos na UTI; (3) OB e não OB e o risco para mortalidade,

VMI, O2 suplementar

(1) 92; (2) Indivíduos consecutivos admitidos em centro hospitalar, sobrepeso ou OB dois terços, maioria homens de meia idade; (3) Sobrepeso, OB e não OB, idade, VMI, aporte de O2, UTI e unidade respiratória semi-intensiva

(1) 124 ; (2) Pacientes internados em UTI SARS-CoV-2, maioria homens, idade $>50$ anos, poucos casos com idade <20 anos; (3) OB e não OB, necessidade de VMI, O2 suplementar
$\mathrm{A}-87 \%$

B $-0 \%$

$\mathrm{A}-87 \%$

$\mathrm{B}-3 \%$

$\mathrm{A}-91 \%$

$\mathrm{B}-3 \%$

Legenda: OB (Obesos), IMC (Índice de Massa Corporal). Fonte: Autores. 
Figura 2 - Resultados dos artigos incluídos na revisão.

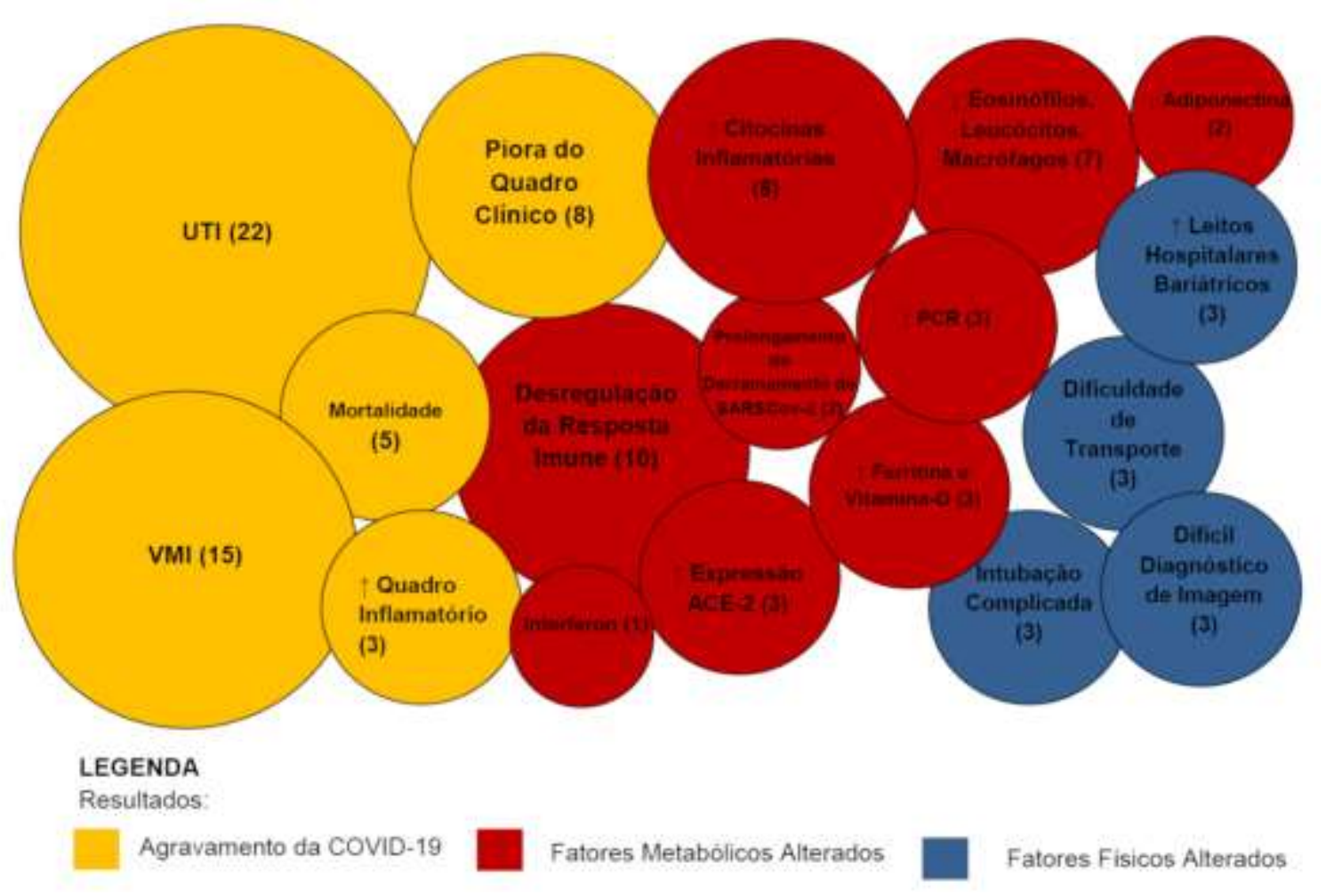

Fonte: Autores.

Em alguns estudos observou-se que pacientes obesos demonstraram piora do quadro inflamatório da doença COVID19 devido a instabilidade imunológica e ao ambiente metabólico desfavorável (Messina et al., 2020; Palaiodimos et al., 2020; Ryan \& Caplice, 2020). Além de maior admissão e permanência em UTI's. Somado a isso, é possível perceber que os índices de internações e complicações no COVID-19 com pacientes que eram obesos, ultrapassou o índice de obesidade presentes na população local do estudo. Ou seja, entre os pacientes com COVID-19 agravada naquela região a maioria eram obesos. (Busetto et al., 2020)

Em relação à resposta imune, estudos mostraram haver uma forte associação entre a obesidade e o estado inflamatório crônico, caracterizada por um conjunto de alterações metabólicas (Belančić, Kresović, \& Rački, 2020). O tecido adiposo não é apenas um local de estoque mas também assume um papel importante na produção de substâncias bioativas, adipocinas, com funções imunológicas como a adipsina, adiponectina, proteína C reativa (PCR), TNF- $\alpha$, leptina, interleucina 6 e IL-1ß. (Inês Grenha et al., 2013). A leptina exerce grande contribuição na resposta imune do organismo, como na proliferação, maturação e diferenciação dos linfócitos, desenvolvimento do timo, produção de citocinas, regulação da apoptose de monócitos e da atividade dos neutrófilos e macrófagos. Indivíduos obesos têm hiperleptinemia o que resulta em uma resistência à leptina, prejudicando as atividades reguladas por ela. Logo, o quadro inflamatório instaurado pela obesidade leva ao agravamento do COVID-19. Na medida em que há uma redução do numero de células funcionalmente ativas do sistema imune, como linfócitos, macrófagos, neutrófilos e eosinófilos.(Luzi \& Radaelli, 2020)

Desta forma, com um aumento de células adiposas ocorre uma alteração na produção de adipocinas inflamatórias, ou seja, quanto maior a quantidade de tecido adiposo maior será a secreção dessas adipocinas pró inflamatórias. A produção de TNF- $\alpha$, PCR, IL-6 e IL-1ß encontra-se elevada enquanto a adiponectina, anti-inflamatória, encontra-se diminuída. Desencadeando a tempestade de citocinas responsáveis pelo grau de inflamação encontrado em pacientes obesos devido ao desequilíbrio imune (Inês Grenha et al., 2013). 
Um estudo levantou a teoria de que se ocorresse uma modificação do regime alimentar para melhorar os níveis de adiponectina poderia se ter um resultado positivo na prevenção da infecção por SARS-Cov-2, além de melhorar o estado dos pacientes já infectados. Haja vista que, o metabolismo do obeso com COVID-19 e a consequente liberação de citocinas próinflamatórias, incluindo interleucina 6 (IL-6) e fator de necrose tumoral (TNF- $\alpha$ ), tem papel significativo na piora do quadro infeccioso diminuindo os nível de adiponectina (Luzi \& Radaelli, 2020; Malavazos, Corsi Romanelli, Bandera, \& Iacobellis, 2020; Messina et al., 2020). No entanto, o efeito positivo proposto pelo trabalho não foi comprovado e os autores chegaram a conclusão que ele é controverso.

Em alguns desfechos, o agravamento da doença COVID-19 em jovens foi atrelado ao excesso de peso, já que esses não possuíam comorbidades associadas além da obesidade (Kass, Duggal, \& Cingolani, 2020; Zhang et al., 2020). Enquanto outros estudos cujo índice de mortalidade era igual para obesos e não obesos, ou até maior para pessoas com peso referencial, havia uma prevalência de pacientes idosos e com comorbidades associadas no grupo de não obesos (Busetto et al., 2020).

Um artigo escrito na China citou as comorbidades associadas à obesidade, com uma tendência ao aumento da disposição para a doença grave de COVID-19 (Engin, Engin \& Engin 2020). Mas, nenhuma associação significativa, nessa população específica, pode ser elucidada com a obesidade grave.

De modo semelhante, o excesso de gordura visceral, é descrita como prejudicial, diminuindo a capacidade funcional pulmonar (Dietz \& Santos-Burgoa, 2020; Luzi \& Radaelli, 2020). Estudos mostram ainda diminuição do volume de reserva expiratório e complacência do sistema, devido às alterações nos movimentos respiratórios. Portanto, os fatores físicos também influenciam no agravamento do COVID-19 em obesos, na medida em que há dificuldade na realização de diagnóstico de imagem, no processo de intubação, transporte e na acessibilidade e acomodação nos leitos (Michalakis \& Ilias, 2020).

Os resultados encontrados na meta-análise referente a frequência combinada dos estudos entre obesos mostraram necessidade de UTI em $27 \%$ dos casos Intervalo de Confiança (IC) $18 \%-37 \%$ ( $\mathrm{I}^{2}=92 \%$ e p< 0,01) com risco relativo (RR) de 1,47 IC 1,29-1,68 (I ${ }^{2}=0 \%$ e p=0,82). VMI foi de 34\% IC 15\%-52\% (I²=96\% e p<0,01) com RR de 1,48 IC de 1,26-1,73 $\left(\mathrm{I}^{2}=0 \%\right.$ e $\left.\mathrm{p}=0,70\right)$. Mortalidade foi de $14 \%$ IC de 5\%-23\% ( $\mathrm{I}^{2}=90 \%$ e $\left.\mathrm{p}<0,01\right)$ com RR de 1,05 IC 0,40-2,76 (I²=81\% e p<0,01) (Tabela 2 e Figura 3). Apesar dos estudos serem heterogênios, isto é terem resultados variados com heterogeneidade clínica, os achados encontrados são significativos.

Tabela 2 - Modelos de meta-análise da frequência de cada desfecho entre obesos e risco relativo (RR) com estimativa do efeito combinado, intervalo de confiança de $95 \%$ (IC) e teste de heterogeneidade ( $\mathrm{I}^{2}$ e p-valor).

\begin{tabular}{lccc}
\hline & $\begin{array}{c}\text { Efeito } \\
\text { combinado }\end{array}$ & IC 95\% & Heterogeneidade \\
\hline Frequência do desfecho em obesos & & & \\
\hline UTI & $27 \%$ & $18 \%-37 \%$ & $\mathrm{I}^{2}=92 \%$ e $\mathrm{p}<0,01$ \\
VMI & $34 \%$ & $15 \%-52 \%$ & $\mathrm{I}^{2}-96 \%$ e $\mathrm{p}<0,01$ \\
Mortalidade & $14 \%$ & $5 \%-23 \%$ & $\mathrm{I}^{2}=90 \%$ e $\mathrm{p}<0,01$ \\
\hline Risco relativo (RR) & & $1,29-1,68$ & $\mathrm{I}^{2}=0 \%$ e $\mathrm{p}=0,82$ \\
\hline UTI & 1,47 & $1,26-1,73$ & $\mathrm{I}^{2}=0 \%$ e $\mathrm{p}=0,70$ \\
VMI & 1,48 & $0,40-2,76$ & $\mathrm{I}^{2}=81 \%$ e $\mathrm{p}<0,01$ \\
Mortalidade & 1,05 & & \\
\hline
\end{tabular}


Figura 3. Forest plot dos modelos de meta-análise do risco relativo (RR) com estimativa do efeito combinado, intervalo de confiança de $95 \%$ (IC) e teste de heterogeneidade ( $\mathrm{I}^{2}$ e p-valor).

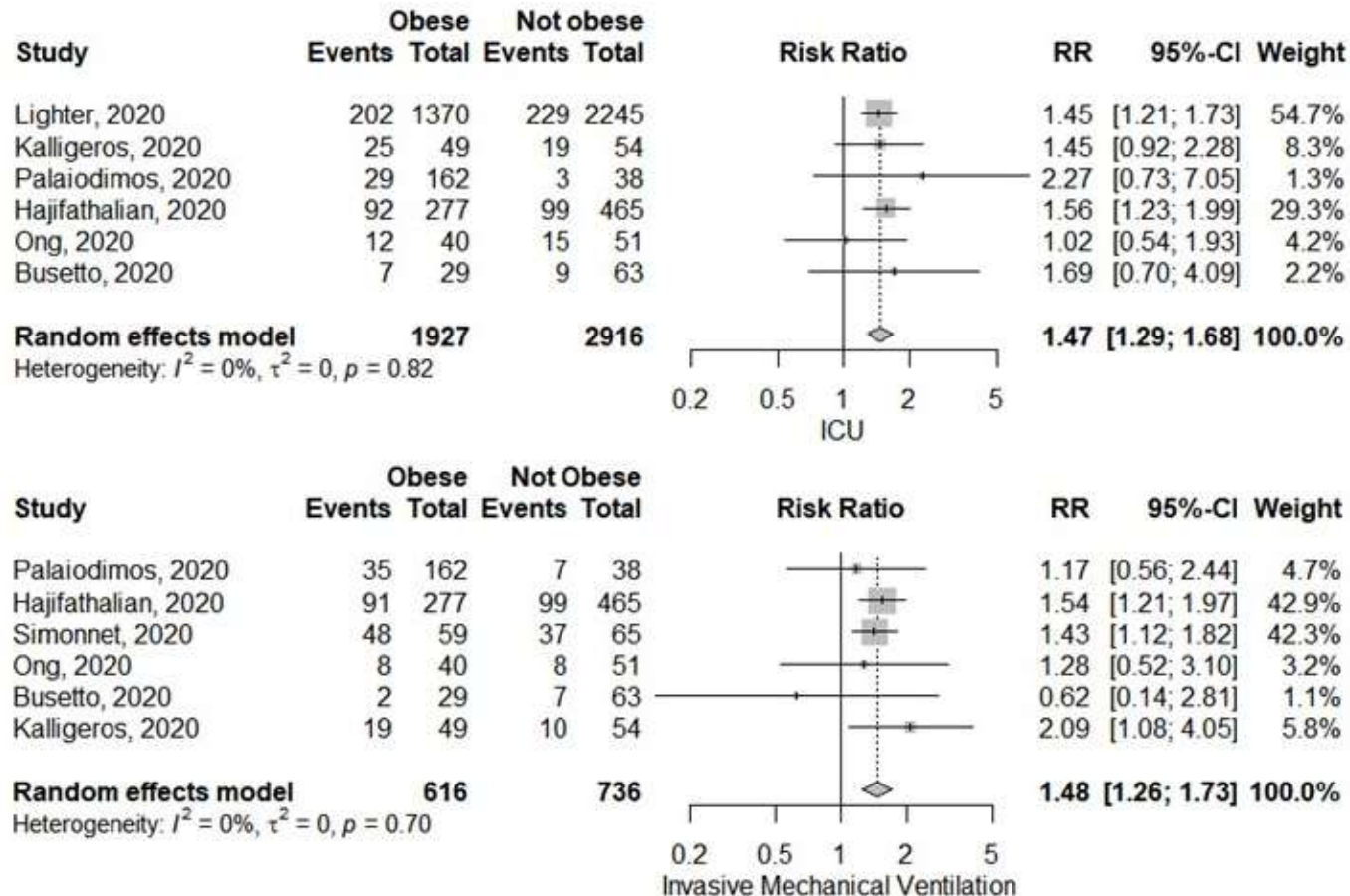

Obese Not Obese
Study $\quad$ Events Total Events Total

$\begin{array}{lrrrr}\text { Kassir, 2020 } & 15 & 33 & 2 & 79 \\ \text { Palaiodimos, 2020 } & 36 & 162 & 12 & 37 \\ \text { Hajifathalian, 2020 } & 22 & 277 & 57 & 465 \\ \text { Ong, 2020 } & 1 & 40 & 3 & 51 \\ \text { Busetto, 2020 } & 2 & 29 & 10 & 63 \\ & & & & \\ \text { Random effects model } & & 541 & & 695 \\ \text { Heterogeneity: } I^{2}=81 \%, \tau^{2}=0.8307, p<0.01 & \end{array}$

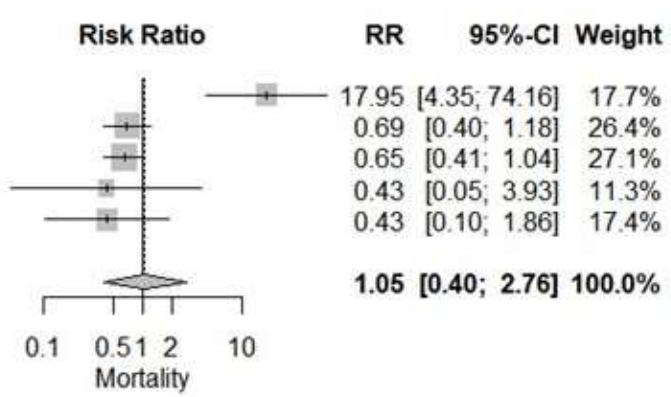

Fonte: Autores.

\section{Discussão}

O COVID-19 é uma doença infecciosa que atingiu proporções globais rapidamente, tendo uma evolução rápida e alta infectividade. Manifesta-se de forma leve ou grave, podendo apresentar desde sintomas brandos como tosse, corisa, cefeléia e febre até Síndrome do Desconforto Respiratório Agudo (SDRA), podendo levar ao óbito (Netto et al., 2020).

Associando a atual transição demográfica da America Latina, em que há um aumento da população em idade ativa no mercado de trabalho, com as condições socioeconômicas e culturais, percebe-se uma diminuição das práticas de exercícios físicos além de uma má alimentação, culminando no aumento das taxas de obesidade nesse público (UOL,2021).

A obesidade como doença crônica e multifatorial está associada a um estado inflamatório crônico, capaz de desenvolver uma série de complicações relacionadas a desregulação hormonal e do sistema imune. Quando essa condição é adicionada ao cenário do novo coronavírus, há um aumento dessas complicações e resultados desfavoráveis (Michalakis \& Ilias, 2020)

Este estudo analisou artigos referentes ao COVID-19 e a obesidade. Os desfechos de necessidade de UTI, VMI e mortalidade foram analisados a partir da meta-análise e da revisão sistemática. Os resultados encontrados foram significativos 
e demonstraram que pacientes obesos apresentaram maior risco de admissão de UTI (27\%) e necessidade de VMI (34\%). Já na mortalidade (14\%), os resultados não foram tão significativos pois na amostra de alguns artigos europeus a prevalência de obesos estava em pacientes jovens enquanto os pacientes não obesos eram em sua maioria idosos, com várias comorbidades e por isso com maior chance de evoluir ao óbito (Moriconi et al., 2020).

Observou-se no presente estudo que dentre os pacientes com COVID-19 admitidos na UTI, mais de 50\% daqueles que necessitaram de VMI eram obesos. Tal fato pode ser explicado em parte porque a massa corporal excessiva favorece complicações físicas em relação ao transporte, acomodação e intubação do paciente obeso (Finer, Garnett \& Bruun,, 2020; Ryan, Ravussin \& Heymsfield, 2020). Em uma revisão abordando o mesmo tema Földi (2020) compilou artigos mostrando que indivíduos obesos precisaram de diferentes formas de suporte respiratório e intubação endotraqueal, além de os mesmos apresentarem maiores dificuldades para realização dos procedimentos de exames complementares.

De maneira geral, os artigos incluídos na presente revisão mostraram que pacientes com IMC mais elevado necessitaram de UTI com maior frequência (Kassir, 2020). Esse achado pode ser explicado tanto pelas reservas pulmonares reduzidas e alterações anatômicas da parede torácica quanto pelas alterações da resposta imunológica (Muscogiuri, Pugliese, Barrea, Savastano \& Colao, 2020). Haja vista que, o tecido adiposo não é apenas um local de estoque mas também tem papel importante na produção de citocinas inflamatórias, que quando em excesso podem agravar o quadro clinico (Dietz \& SantosBurgoa, 2020; Petrakis et al., 2020).

A revisão sistemática de Malik (2020), relatou haver um associação mais significativa entre a obesidade em pacientes idosos e com COVID-19. Entretanto nos artigos incluídos nesta revisão não houve distinção de faixa etária entre os pacientes obesos e com agravamento de COVID-19, pelo contrário, houve uma prevalência de obesidade em pacientes mais jovens, principalmente nos estudos feitos nos EUA, em que a obesidade populacional está acima da média mundial (Watanabe et al., 2020).

Siqueira (2020) descreve correlação entre aumento do IMC, especialmente acima de $30 \mathrm{~kg} / \mathrm{m}^{2}$, com piores desfechos em pacientes infectados com o novo coronavírus. Essa relação foi semelhante a encontrada no presente estudo de revisão, dado que os artigos incluídos apontaram maior gravidade das manifestações clínicas do coronavírus em pacientes obesos. Entretanto, não foi analisado a obesidade apenas de acordo com os valores padrões (OMS) de IMC ( $\left.>30 \mathrm{Kg} / \mathrm{m}^{2}\right)$, pois em dois estudos orientais, levou-se em conta as diferenças étnicas da população. Nesses artigos os pacientes considerados obesos apresentavam IMC superior a $25 \mathrm{Kg} / \mathrm{m}^{2}$ (Kassir, 2020; Ong, Young, Leo \& Lye, 2020).

Ainda, observou-se uma variação significativa em relação ao contingente de pacientes abordados pelos estudos incluídos nessa revisão. Isto é, o tamanho da amostra nos trabalhos foi de 53 pacientes (Zhang et al., 2020) a 3615 (Lighter et al., 2020). No entanto, apesar da amostra ser pequena, na maioria dos estudos, a qualidade geral não foi prejudicada. Uma análise semelhante foi encontrada na revisão de Tamara (2020) na qual apesar do estudo apresentar uma amostragem menor (Simonnet et al., 2020), a qualidade dos seus dados é melhor.

A avaliação de qualidade apresentou variações, mas a maioria dos estudos tiveram escore superior a 75\%. Percebe-se também que houve uma grande abrangência de países, bem como estudos multicêntricos e unicêntricos. Além disso, a heterogeneidade dos artigos em geral mostrou-se significativa.

Na meta-análise realizada neste trabalho, a seleção e extração dos dados quantitativos referentes ao desfecho UTI de dois estudos (Kalligeros et al., 2020; Lighter et al., 2020) diferiram de outra revisão sistemática (Földi et al., 2020). Pois a meta-análise realizada por Földi (2020) não incluiu o total da amostra sugerindo possível viés nos resultados obtidos.

A presente revisão apresentou limitações, dado que nos estudos selecionados, houve indisponibilidade da totalidade de informações em relação aos fatores metabólicos, características dos pacientes (idade, IMC e outras comorbidades), especificação se o estudo era multicêntrico ou unicêntrico, avaliação inadequada e incompleta dos dados extraídos dos 
prontuários ou apresentação deficiente dos mesmos. Além disso, não foi possível fazer estratificação maior entre as classes de obesidade. Outra limitação digna de nota, é que poucos estudos apresentaram dados quantitativos, diante desse fato a análise de viés de publicação nos estudos não pôde ser realizada pois o gráfico de funil e teste de Egger (metodologias adequadas para este fim) devem ser aplicados quando há, pelo menos, sete estudos com dados disponíveis. No caso da presente revisão, os modelos foram construídos com 5-6 artigos, demonstrando que a evidência aqui relatada ainda precisa de mais estudos originais para que tenha mais robustez.

A COVID-19 foi uma emergência de saúde pública em 2019-2020 e até a data da presente revisão, havia uma quantidade considerável de pesquisas em andamento e sendo publicadas a todo momento. Por isso, considera-se que o nível de evidência das associações pode apresentar mudanças até a versão final de nossa revisão.

\section{Conclusão}

A presente revisão sistemática avaliou a relação da COVID-19 em pacientes obesos. Analisando os desfechos necessidade de UTI, VMI e mortalidade conclui-se que a obesidade é um fator preditor para o agravamento da COVID-19 em todos os continentes. Dessa forma, indivíduos obesos necessitam de uma abordagem precoce bem como um atendimento especial em caso de contaminação pelo vírus, haja vista que nesses pacientes há um ambiente hormonal desfavorável, que desregula o sistema imune e desencadeia a piora do quadro clínico.

\section{Referências}

Balduzzi, S., Rücker, G., S. G. (2019). How to perform a meta-analysis with R: a practical tutorial. Evidence-Based Mental Health, 153-160.

Belančić, A., Kresović, A., \& Rački, V. (2020). Potential pathophysiological mechanisms leading to increased COVID-19 susceptibility and severity in obesity. In Obesity Medicine (Vol. 19). Elsevier Ltd. https://doi.org/10.1016/j.obmed.2020.100259

Brasil. Ministério da Saúde. Secretaria de Ciência, T. e I. E. D. de C. e T. (2014). Diretrizes metodológicas: elaboração de revisão sistemática e metanálise de estudos observacionais comparativos sobre fatores de risco e prognóstico. www.saude.gov.br

Busetto, L., Bettini, S., Fabris, R., Serra, R., Dal Pra', C., Maffei, P., Rossato, M., Fioretto, P., \& Vettor, R. (2020). Obesity and COVID-19: an Italian snapshot. Obesity. https://doi.org/10.1002/oby.22918

de Oliveira, A. C., Lucas, T. C., \& Iquiapaza, R. A. (2020). What has the covid-19 pandemic taught us about adopting preventive measures? Texto e Contexto Enfermagem, 29, 1-15. https://doi.org/10.1590/1980-265X-TCE-2020-0106

de Siqueira, J. V. V., Almeida, L. G., Zica, B. O., Brum, I. B., Barceló, A., \& de Siqueira Galil, A. G. (2020). Impact of obesity on hospitalizations and mortality, due to COVID-19: A systematic review. In Obesity Research and Clinical Practice. Elsevier Ltd. https://doi.org/10.1016/j.orcp.2020.07.005

Dietz, W., \& Santos-Burgoa, C. (2020). Obesity and its Implications for COVID-19 Mortality. In Obesity (Vol. 28, Issue 6, p. 1005). Blackwell Publishing Inc. https://doi.org/10.1002/oby.22818

Engin, A. B., Engin, E. D., \& Engin, A. (2020). Two important controversial risk factors in SARS-CoV-2 infection: Obesity and smoking. In Environmental Toxicology and Pharmacology (Vol. 78). Elsevier B.V. https://doi.org/10.1016/j.etap.2020.103411

Estudo aponta que 60\% dos adultos da América Latina ganharam peso na pandemia - 07/05/2021 - UOL Economia. (2021). https://economia.uol.com.br/noticias/efe/2021/05/07/estudo-aponta-que-60-dos-adultos-da-america-latina-ganharam-peso-na-pandemia.htm

Finer, N., Garnett, S. P., \& Bruun, J. M. (2020). COVID-19 and obesity. Clinical Obesity, 10(3). https://doi.org/10.1111/cob.12365

Földi, M., Farkas, N., Kiss, S., Zádori, N., Váncsa, S., Szakó, L., Dembrovszky, F., Solymár, M., Bartalis, E., Szakács, Z., Hartmann, P., Pár, G., Erőss, B., Molnár, Z., Hegyi, P., \& Szentesi, A. (2020). Obesity is a risk factor for developing critical condition in COVID-19 patients: A systematic review and meta-analysis. Obesity Reviews, 21(10). https://doi.org/10.1111/obr.13095

Hajifathalian, K., Kumar, S., Newberry, C., Shah, S., Fortune, B., Krisko, T., Ortiz-Pujols, S., Zhou, X. K., Dannenberg, A. J., Kumar, R., \& Sharaiha, R. Z. (2020). Obesity is associated with worse outcomes in COVID-19: Analysis of Early Data From New York City. Obesity. https://doi.org/10.1002/oby.22923

Inês Grenha, A., Alves, F., Ribeiro, F., \& Cavaco, T. (2013). Obesidade e imunodepressão: Factos e números.

Kalligeros, M., Shehadeh, F., Mylona, E. K., Benitez, G., Beckwith, C. G., Chan, P. A., \& Mylonakis, E. (2020). Association of Obesity with Disease Severity Among Patients with Coronavirus Disease 2019. Obesity, 28(7), 1200-1204. https://doi.org/10.1002/oby.22859

Kass, D. A., Duggal, P., \& Cingolani, O. (2020). Obesity could shift severe COVID-19 disease to younger ages. In The Lancet (Vol. 395, Issue 10236, pp. 1544-1545). Lancet Publishing Group. https://doi.org/10.1016/S0140-6736(20)31024-2 
Kassir, R. (2020). Risk of COVID-19 for patients with obesity. In Obesity Reviews (Vol. 21, Issue 6). Blackwell Publishing Ltd. https://doi.org/10.1111/obr.13034

Lighter, J., Phillips, M., Hochman, S., Sterling, S., Johnson, D., Francois, F., \& Stachel, A. (2020). Obesity in Patients Younger Than 60 Years Is a Risk Factor for COVID-19 Hospital Admission. In Clinical infectious diseases : an official publication of the Infectious Diseases Society of America (Vol. 71, Issue 15, pp. 896-897). NLM (Medline). https://doi.org/10.1093/cid/ciaa415

Luiz Do Prado, W., Lofrano, M. C., Oyama, L. M., \& Dâmaso, A. R. (2009). Artigo de revisão Obesidade e Adipocinas Inflamatórias: Implicações Práticas para a Prescrição de Exercício Obesity and Inflammatory Adipokines: Practical Implications for Exercise Prescription Endereço para correspondência. In Rev Bras Med Esporte (Vol. 15).

Luzi, L., \& Radaelli, M. G. (2020). Influenza and obesity: its odd relationship and the lessons for COVID-19 pandemic. Acta Diabetologica, 57(6), 759-764. https://doi.org/10.1007/s00592-020-01522-8

Malavazos, A. E., Corsi Romanelli, M. M., Bandera, F., \& Iacobellis, G. (2020). Targeting the Adipose Tissue in COVID-19. Obesity. https://doi.org/10.1002/oby. 22844

Malik, V. S., Ravindra, K., Attri, S. V., Bhadada, S. K., \& Singh, M. (2020). Higher body mass index is an important risk factor in COVID-19 patients: a systematic review and meta-analysis. Environmental Science and Pollution Research, 1-9. https://doi.org/10.1007/s11356-020-10132-4

Messina, G., Polito, R., Monda, V., Cipolloni, L., Di Nunno, N., Di Mizio, G., Murabito, P., Carotenuto, M., Messina, A., Pisanelli, D., Valenzano, A., Cibelli, G., Scarinci, A., Monda, M., \& Sessa, F. (2020). Functional role of dietary intervention to improve the outcome of COVID-19: A hypothesis of work. International Journal of Molecular Sciences, 21(9). https://doi.org/10.3390/ijms21093104

Michalakis, K., \& Ilias, I. (2020). SARS-CoV-2 infection and obesity: Common inflammatory and metabolic aspects. Diabetes and Metabolic Syndrome: Clinical Research and Reviews, 14(4), 469-471. https://doi.org/10.1016/j.dsx.2020.04.033

Moriconi, D., Masi, S., Rebelos, E., Virdis, A., Manca, M. L., De Marco, S., Taddei, S., \& Nannipieri, M. (2020). Obesity prolongs the hospital stay in patients affected by COVID-19, and may impact on SARS-COV-2 shedding. Obesity Research \& Clinical Practice, 14(3), 205-209. https://doi.org/10.1016/j.orcp.2020.05.009

Muscogiuri, G., Pugliese, G., Barrea, L., Savastano, S., \& Colao, A. (2020). Obesity: The "Achilles heel" for COVID-19? Metabolism: Clinical and Experimental, 108. https://doi.org/10.1016/j.metabol.2020.154251

Netto, F. de F., Benassi, G., Nogueira, L. S., Ciriaco, E. M., Santos, G. C. dos, Lopes, H., \& Cabral, G. (2020). Novo coronavírus e a COVID-19 - NUTEAD. https://ead.uepg.br/site/moocs/218

Obesidade e excesso de peso. (2020). https://www.who.int/news-room/fact-sheets/detail/obesity-and-overweight

Ong, S. W. X., Young, B. E., Leo, Y. S., \& Lye, D. C. (2020). Association of higher body mass index (BMI) with severe coronavirus disease 2019 (COVID19) in younger patients. Clinical Infectious Diseases: An Official Publication of the Infectious Diseases Society of America. https://doi.org/10.1093/cid/ciaa548

ONU vê "epidemia de obesidade” na América Latina e Caribe | As Nações Unidas no Brasil. (2019, July 9). https://brasil.un.org/pt-br/83657-onu-veepidemia-de-obesidade-na-america-latina-e-caribe

OPAS/OMS Brasil - Folha informativa - COVID-19 (doença causada pelo novo coronavírus). (2020). OPAS/OMS Brasil - Início. https://www.paho.org/bra/

Palaiodimos, L., Kokkinidis, D. G., Li, W., Karamanis, D., Ognibene, J., Arora, S., Southern, W. N., \& Mantzoros, C. S. (2020). Severe obesity is associated with higher in-hospital mortality in a cohort of patients with COVID-19 in the Bronx, New York. Metabolism: Clinical and Experimental, 108. https://doi.org/10.1016/j.metabol.2020.154262

Petrakis, D., Margină, D., Tsarouhas, K., Tekos, F., Stan, M., Nikitovic, D., Kouretas, D., Spandidos, D. A., \& Tsatsakis, A. (2020). Obesity - a risk factor for increased COVID-19 prevalence, severity and lethality (Review). In Molecular Medicine Reports (Vol. 22, Issue 1, pp. 9-19). Spandidos Publications. https://doi.org/10.3892/mmr.2020.11127

Ryan, D. H., Ravussin, E., \& Heymsfield, S. (2020). COVID 19 and the Patient with Obesity - The Editors Speak Out. In Obesity (Vol. 28, Issue 5, p. 847). Blackwell Publishing Inc. https://doi.org/10.1002/oby.22808

Ryan, P. M. D., \& Caplice, N. M. (2020). Is Adipose Tissue a Reservoir for Viral Spread, Immune Activation, and Cytokine Amplification in Coronavirus Disease 2019? In Obesity (Vol. 28, Issue 7, pp. 1191-1194). Blackwell Publishing Inc. https://doi.org/10.1002/oby.22843

Simonnet, A., Chetboun, M., Poissy, J., Raverdy, V., Noulette, J., Duhamel, A., Labreuche, J., Mathieu, D., Pattou, F., Jourdain, M., Caizzo, R., Caplan, M., Cousin, N., Duburcq, T., Durand, A., El kalioubie, A., Favory, R., Garcia, B., Girardie, P., ... Verkindt, H. (2020). High prevalence of obesity in severe acute respiratory syndrome coronavirus-2 (SARS-CoV-2) requiring invasive mechanical ventilation. Obesity. https://doi.org/10.1002/oby.22831

Singh, J. (2010). Mendeley: A free research management tool for desktop and web. In Journal of Pharmacology and Pharmacotherapeutics (Vol. 1, Issue 1, pp. 62-63). Wolters Kluwer -- Medknow Publications. https://doi.org/10.4103/0976-500X.64539

Tamara, A., \& Tahapary, D. L. (2020). Obesity as a predictor for a poor prognosis of COVID-19: A systematic review. Diabetes and Metabolic Syndrome: Clinical Research and Reviews, 14(4), 655-659. https://doi.org/10.1016/j.dsx.2020.05.020

Watanabe, M., Risi, R., Tuccinardi, D., Baquero, C. J., Manfrini, S., \& Gnessi, L. (2020). Obesity and SARS-CoV-2: a population to safeguard. In Diabetes/Metabolism Research and Reviews. John Wiley and Sons Ltd. https://doi.org/10.1002/dmrr.3325

Zhang, F., Xiong, Y., Wei, Y., Hu, Y., Wang, F., Li, G., Liu, K., Du, R., Wang, C. Y., \& Zhu, W. (2020). Obesity predisposes to the risk of higher mortality in young COVID-19 patients. Journal of Medical Virology. https://doi.org/10.1002/jmv.26039 